\title{
Real-Time Building Energy Simulation Using EnergyPlus and the Building Controls Test Bed
}

Xiufeng Pang, Prajesh Bhattachayra, Zheng O’Neill, Philip Haves, Michael Wetter and Trevor Bailey

November 2011 


\section{DISCLAIMER}

This document was prepared as an account of work sponsored by the United States Government. While this document is believed to contain correct information, neither the United States Government nor any agency thereof, nor the Regents of the University of California, nor any of their employees, makes any warranty, express or implied, or assumes any legal responsibility for the accuracy, completeness, or usefulness of any information, apparatus, product, or process disclosed, or represents that its use would not infringe privately owned rights. Reference herein to any specific commercial product, process, or service by its trade name, trademark, manufacturer, or otherwise, does not necessarily constitute or imply its endorsement, recommendation, or favoring by the United States Government or any agency thereof, or the Regents of the University of California. The views and opinions of authors expressed herein do not necessarily state or reflect those of the United States Government or any agency thereof or the Regents of the University of California. 


\title{
REAL-TIME BUILDING ENERGY SIMULATION USING ENERGYPLUS AND THE BUILDING CONTROLS VIRTUAL TEST BED
}

\author{
Xiufeng Pang ${ }^{1}$, Prajesh Bhattacharya ${ }^{1}$, Zheng O’Neill ${ }^{2}$, Philip Haves ${ }^{1}$, Michael Wetter ${ }^{1}$, and \\ Trevor Bailey ${ }^{2}$ \\ ${ }^{1}$ Lawrence Berkeley National Laboratory, Berkeley, CA, USA \\ ${ }^{2}$ United Technologies Research Center, East Hartford, CT, USA
}

\begin{abstract}
Most commercial buildings do not perform as well in practice as intended by the design and their performances often deteriorate over time. Reasons include faulty construction, malfunctioning equipment, incorrectly configured control systems and inappropriate operating procedures (Haves et al., 2001, Lee et al., 2007). To address this problem, the paper presents a simulation-based whole building performance monitoring tool that allows a comparison of building actual performance and expected performance in real time. The tool continuously acquires relevant building model input variables from existing Energy Management and Control System (EMCS). It then reports expected energy consumption as simulated of EnergyPlus. The Building Control Virtual Test Bed (BCVTB) is used as the software platform to provide data linkage between the EMCS, an EnergyPlus model, and a database. This paper describes the integrated realtime simulation environment. A proof-of-concept demonstration is also presented in the paper.
\end{abstract}

\section{INTRODUCTION}

EnergyPlus (US DOE, 2010; Crawley et al., 2001) is a detailed first principles based simulation tool that calculates the building heating and cooling loads, and disaggregates energy end uses and other variables required for a comprehensive comparison of simulated and measured performance. Conventionally, EnergyPlus is used for off-line building energy simulation analyze design for new construction and retrofit, size HVAC equipment, and model energy and water use in buildings.

With the increasing need to improve building performance, the use of simulation to assess the actual performance of buildings is starting to gain more attention (Haves et al. 2001, Liu et al. 2003, Ramirez et al. 2005). This paper describes a proofof-concept implementation of EnergyPlus in a realtime application, which represents a step towards the development and deployment of simulation-based building performance assessment techniques.

Real-time building simulation, as opposed to off-line building simulation, refers to the use of a building model whose simulation time is synchronized with real time, as represented by the computer clock. Updated values of the input variables are acquired dynamically at each step-time. With the wide deployment of Energy Management and Control Systems (EMCS) in buildings and the development of open protocols such as BACnet, the sensor and control signal information from various component and systems in a building is more acccessible (Salsbury et al. 2000). This makes it possible to acquire the real-time EnergyPlus dynamic input variables from the EMCS including but not limited to weather data, operation schedules, control set points. However, the EMCS does not normally have all the necessary model input variables that are needed for real-time simulation, e.g. solar radiation, wind speed and direction and additional instrumentation is required to accomadate these needs.

The Building Controls Virtual Test Bed (BCVTB), recently developed by Lawrence Berkeley National Laboratory (LBNL), has provided a platform to synchronize EnergyPlus simulation time to real-time and exchange data with EMCS in the real-time mode as well (Wetter, 2010; Nouidui et al., 2011). It is an extension of Ptolemy II, a software environment for heterogeneous modeling and simulation. Ptolemy II is a free open-source software developed at the University of California, Berkeley.

This paper describes the integrated real-time simulation environment as well as the additional instrumentation required by the real-time simulation. A proof-of-concept demonstration is then described.

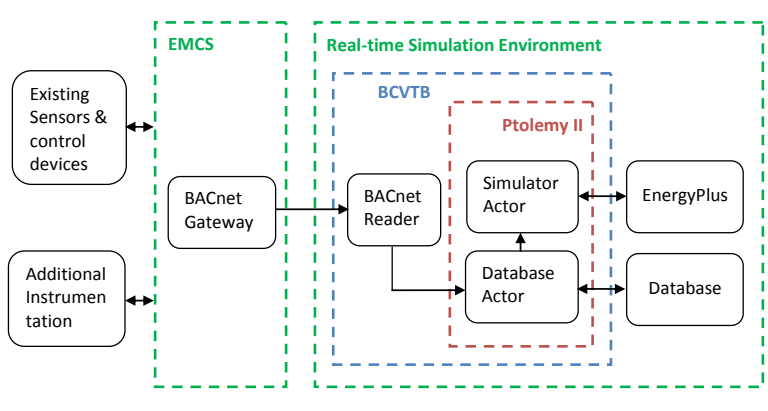

Figure 1 Overall system architecture

\section{SYSTEM INFRASTRUCTURE}

Figure 1 shows the overall system architecture. It consists of two sub-systems: (i) the EMCS that serves as the data acquisition system and (ii) the realtime simulation environment that integrates the EnergyPlus simulation, database and the data 
acquisition system, and synchronizes the simulation to real time. The sub-systems reside in two different computers connected using a Local Area Network (LAN). The following two sections describe these sub-systems in detail.

\section{Instrumentation and Data acquisition}

EnergyPlus model inputs consist of a full description of the building (location, geometry, materials, window type etc.), its usage and the HVAC system description. The weather data is specified separately from the general input file when the simulation is launched. All these input data can be categorized into two types: parameters and variables. Parameters are independent of time and remain constant during the simulation, e.g. building geometry and HVAC equipment nominal capacity etc. Variables may change during the simulation, e.g. weather conditions, control setpoints etc. Some of the variables can be specified in the EnergyPlus input file using schedules, e.g. HVAC equipment operational and control setpoints, while some of the variables can only be obtained from external data source at each step time, e.g. weather conditions, including outdoor dry bulb temperature and relative humidity, wind speed and direction, and direct normal and diffuse solar radiation. For real-time applications, the external variables need to be updated at each step time.

Modern buildings are typically equipped with EMCS, which can provide a number of these variables. However, except for outdoor dry bulb and relative humidity, weather variables are not usually measured by the EMCS. Supplementary instrumentation, usually hosted by the EMCS, is needed for these nontypical measurements.

\section{EMCS Integration}

Additional sensors are typically required to provide, all the variables that are needed by EnergyPlus. In order to make EMCS measurements accessible to EnergyPlus, a suitable communication protocol is needed. BACnet is used in this study due to its wide acceptance. In cases where the EMCS uses a proprietary protocol, a BACnet gateway is required. The BCVTB contains two actors that can read from and write to BACnet devices. The read function aquires the relevant model input variables while the write function provides a hardware-in-the-loop control capability. Both actors use a configuration file to specify the BACnet devices, object types and property identifiers. The detailed procedures to create these configuration files and to use these two actors are described in the BCVTB manual 2011.

\section{EnergyPlus Integration}

Figure 2 shows the connection between the EnergyPlus and the BCVTB. The simulator actor in the BCVTB links to the external interface in the EnergyPlus. In the external interface, the input/output signals that are exchanged between the BCVTB and the EnergyPlus are mapped to EnergyPlus objects

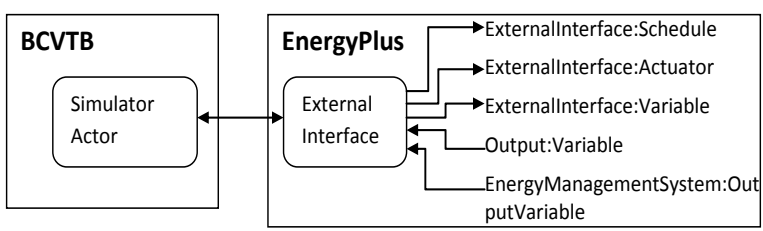

Figure 2 EnergyPlus integration in the BCVTB

(Wetter, 2010). The external interface takes three types of inputs from the BCVTB through three objects:

- ExternalInterface:Schedule,

- ExternalInterface:Actuator,

- ExternalInterface:Variable.

When the BCVTB passes a value to the ExternalInterface:Schedule object, it creates a new schedule. The other two objects are used in the same way as the EnergyPlus Energy Management System (EMS) actuators and variables, except that their numerical values are obtained from the BCVTB at the beginning of each zone time step. The EMS is a newly released feature of EnergyPlus to provide userdefined supervisory control capabilities. It can read a variety of "sensor" data and use this data to direct various types of control actions (USDOE 2010). The actuator objects overwrite various input parameters, such as weather data and set-points and internal calculated variables, such as fractional window opening. Any EnergyPlus Output:Variable or EnergyManagementSystem:OutputVariable can be sent to the BCVTB at each zone time step.

\section{Database Integration}

PostgreSQL was chosen as the database program. A Java-based API was built for applications to communicate easily with the database. In Figure 3 and Figure 4, at the extremme right hand end, there are PtolemyII actors named "database" and "database2", respectively. Both of these are PtolemyII SystemCommand actors and execute a wrapper tool around the database API, in order to send data to the database. At the extreme left hand end of Figure 4, there is an actor called "SQL Statement" which is used to query data from the database.

\section{PROOF-OF-CONCEPT DEMONSTRATION}

A proof-of-concept demonstration has been conducted at a two-storey building located in the Chicago, IL area. The gross area of this building is approximately $70,000 \mathrm{ft}^{2}\left(6503 \mathrm{~m}^{2}\right)$. About $80 \%$ of the floor area serves as drill hall for personnel training and ceremonies. The rest of the building is used for administration and is lightly occupied.

There are two 100 ton $(351.7 \mathrm{~kW})$ air-cooled chillers and associated chilled water pumps providing cooling for whole building. Steam for heating is supplied by a campus-wide distribution system and 
converted to hot water locally. The Drill hall is supplied by two single-zone Variable Air Volume (VAV) Air Handling Units (AHU). The office area is served by one VAV AHU with VAV terminal units. There is a classroom in the building, which is served by a single-zone VAV AHU.

\section{Instrumentation}

A commercial EMCS is installed in the demonstration building. In order to accommodate the needs of real-time energy simulation, additional sensors were installed to provide the measurements for outdoor air dry bulb and relative humidity, wind speed and direction, direct normal solar radiation and diffuse solar radiation. High quality sensors were used to minimize the impacts from the weather on the model output uncertainties. In order to compare the simulation results with the actual performance of the building sub-systems such as lighting, plug-loads and chillers, dedicated electrical power sub-meters were installed to measure the lighting power, plug load power and chiller power. The requirements for the sensor accuracies were taken from Gillespie et al. (2007).

\section{System Setup}

A whole-building EnergyPlus simulation model representing the existing performance of the envelope, the HVAC system, the lighting system and the control system was created. The model was then calibrated based on the information collected from design documents as well as EMCS trending data from April to July 2010. Since the EMCS uses a proprietary communication protocol, a BACnet server was installed on top of the EMCS so that the BACnet interface included in the BCVTB can communicate with the EMCS. The BCVTB was installed in a separate computer from the one running EMCS. The real-time simulation is launched by starting the BCVTB through the Graphical User Interface (GUI) or through the console. The latter allows use of the BCVTB in an automated workflow or in a window-less system.

There are two processes running in parallel. The integration of the BACnet reader using the Ptolemy II GUI is illustrated in Figure 3. The two key elements are the BACnet actor and the database actor. The BACnet actor acquires the EMCS data in five-minute interval. The StringArrayToDoubleArray actor is used to convert the BACnet actor output data from string to double. The database actor receives the double data and sends the data to the database. The actors enclosed by the red dash line are used to calculate the outdoor dew point taking the inputs of outdoor dry bulb and relative humidity.

The integration of the EnergyPlus simulation program using the Ptolemy II GUI is shown in Figure 4. The EnergyPlus simulator establishes the communication between the BCVTB and the calibrated EnergyPlus model. The EnergyPlus simulation time step is 15 minutes. At each time step, the EnergyPlus simulator receives the weather data, i.e. the outdoor air dry bulb temperature, the outdoor relative humidity, the outdoor dew point, the wind speed and direction, the direct normal solar radiation, and the diffuse solar radiation as inputs through the SQLStatement actor, as dynamic inputs and advances the EnergyPlus model by one step time.

Since the BACnet read process uses a 5 minute timestep while EnergyPlus simulation uses 15 minutes, the inputs to the EnergyPlus simulation are averages of the measurements over the 15 minutes. Once the simulation is finished for each time-step, the results are passed to the database. The BCVTB time-step and the run period are specified before starting the simulation, and should match the EnergyPlus timestep.

Since EnergyPlus can only specify the begin day and month, unless the simulation starts right at mid-night, a delay-start of the simulation is needed to ensure that the EnergyPlus simulation synchronizes to wall clock time. In the PtolemyII GUI, synchronization of the simulation time and real-time is achieved by checking a check box, as shown in Figure 5. Then EnergyPlus runs the model and sends the required output data back to the BCVTB.

If the EnergyPlus simulation and the BACnet read process use the same time step, an alternative is that the EnergyPlus simulator can obtain the input variables directly from the BACnet actor instead of the database.

The BCVTB version 0.6.0 is used in the demonstration. A customized EnergyPlus based on version 5.0 is used in this study to provide the actuators for the weather data. This feature is available in the latest release of EnergyPlus 6.0.

\section{Results and Discussion}

The tool chain has been used since July 2010. The first test was to validate the real-time data acquisition process. The existing EMCS has about 1,200 control points including both physical points and virtual points such as control set points etc. About 700 control points that are relevant to the demonstration have been broadcasted through the BACnet server. The BACnet reader in the BCVTB reads these data points and sends to the database. Several manual checks have been conducted by exporting the data from the database to a spreadsheet and comparing to the trending data in the EMCS over one month period. The comparison shows that the data stored in the database match the trend data in the EMCS exactly.

To validate the real-time EnergyPlus operation, a customized EnergyPlus weather file was created using the actual weather data stored in the database. The real-time EnergyPlus simulation results stored in the database were then compared to that of the offline EnergyPlus simulation using the customized 
weather file. As can be seen from Figure 6, the simulation results for the building total electric power agree well with each other.

The real-time whole building energy performance is compared to the model prediction as shown in Figure 7. The differences between the simulated and measured performance were highlighted. The highlighted areas enclosed by the oval dash lines represent the differences occurred during occupied hours while that enclosed by the rectangle dash lines represent the differences occurred during unoccupied hours. Further analysis of the electric power indicated that different chiller operation strategies were the cause of the considerable performance deviations during occupied hours, as shown in Figure 8. The chiller was scheduled to be off after Oct 15 in EnergyPlus model as intended, while in reality, chiller ON/OFF is only controlled by the outdoor air temperature. Simulation shows that free cooling is sufficient to handle the building cooling load. The lights left on overnight was the cause of the deviations during unoccupied hours, as shown in Figure 9.

\section{CONCLUSION}

EnergyPlus is a whole building simulation program that is traditionally used for off-line building energy analysis. A tool for implementing real-time EnergyPlus simulation has been demonstrated in proof-of-concept form. It provides a means to compare actual building performance to design intent or other benchmarks. The demonstration shows the potential of such a tool for supporting whole building performance assessment.

Future work includes testing the implementation using shorter time step, e.g. one minute, fixing EnergyPlus run control and incorporating more communication protocols. The proof-of-concept demonstration used 15 minutes as the simulation time step which is not adequate to represent the system dynamics, e.g. the HVAC equipment short cycling, the plug load equipment on/off etc. Therefore, a validation of the tool using one minute simulation time step is critical. To handle the leap year and to start the real-time simulation at any given time need to be addressed in the EnergyPlus run control.

\section{ACKNOWLEDGEMENT}

This project is a collaboration between LBNL and the United Technologies Research Center and is funded by the ESTCP program of the US Department of Defense and by the Assistant Secretary for Energy Efficiency and Renewable Energy, Office of Building Technology, State and Community Programs of the U.S. Department of Energy under Contract No. DE-AC02-05CH11231. Views, opinions, and/or findings contained in this paper are those of the authors and should not be construed as an official Department of Defense position or decision unless so designated by other official document.

\section{REFERENCES}

BCVTB manual. 2011. http://simulationresearch.lbl. gov/bcvtb/releases/1.0.0/doc/manual/index.xhtm 1

Crawley, D.B., Lawrie, L.K., Winkelmann, F.C., Buhl, W.F., Huang, Y. J., Pederse,n C.O., Strand, R.K., Liesen, R.J., Fisher, D. E., Witte, M.J. and Glazer, J. 2001. EnergyPlus: creating a new-generation building energy simulation program. Energy and Buildings, 33: 319-331.

Haves, P., Salsbury, T., Claridge, D. and Liu M. 2001. Use of Whole Building Simulation in OnLine Performance Assessment: Modeling and Implementation Issues. Proceedings of $7^{\text {th }}$ International IBPSA Conference Building Simulation 2001, Aug 13-15, 2001, Rio de Janeiro.

Lee, S. Uk, Painter, F. L. and Claridge, D. E. 2007. Whole-Building Commercial HVAC System Simulation for Use in Energy Consumption Fault Detection. ASHRAE Transactions, v113, part2: 52-61.

Liu, M., Claridge, D. E., Bensouda, N., Heinemeier, K., Lee, S. Uk, Wei, G., and Mathew, P. 2003. Manual of Procedures for Calibrating Simulations of Building Systems. Berkeley, CA: Lawrence Berkeley National Laboratory.

Nouidui, T.S., Wetter, M., Li,Z., Pang, X., Bhattacharya,P. and Haves, P. BACnet and analog/digital interfaces of the Building Controls Virtual Test Bed. Proceedings of $12^{\text {th }}$ International IBPSA Conference Building Simulation 2011, Nov 14-17, 2011, Sydney, Australia

Ramirez, R., Sebold, F., Mayer, T., Ciminelli, M., and Abrishami, M. 2005. A building simulation palooza: the California CEUS project and DrCEUS. Proceedings of Building Simulation 2005, Montreal, Canada. IBPSA, 2005.

Salsbury, T. and Diamond, R. 2000. Performance validation and energy analysis of HVAC systems using simulation. Energy and Buildings, 32:5-17.

US DOE. 2010. EnergyPlus Documentation. http://apps1.eere.energy.gov/buildings/energyplu s/energyplus_documentation.cfm

Wetter, M. 2010. Co-simulation of Building Energy and Control Systems with the Building Controls Virtual Test Bed. Journal of Building Performance Simulation, First published on: 05 November 2010 (iFirst) 


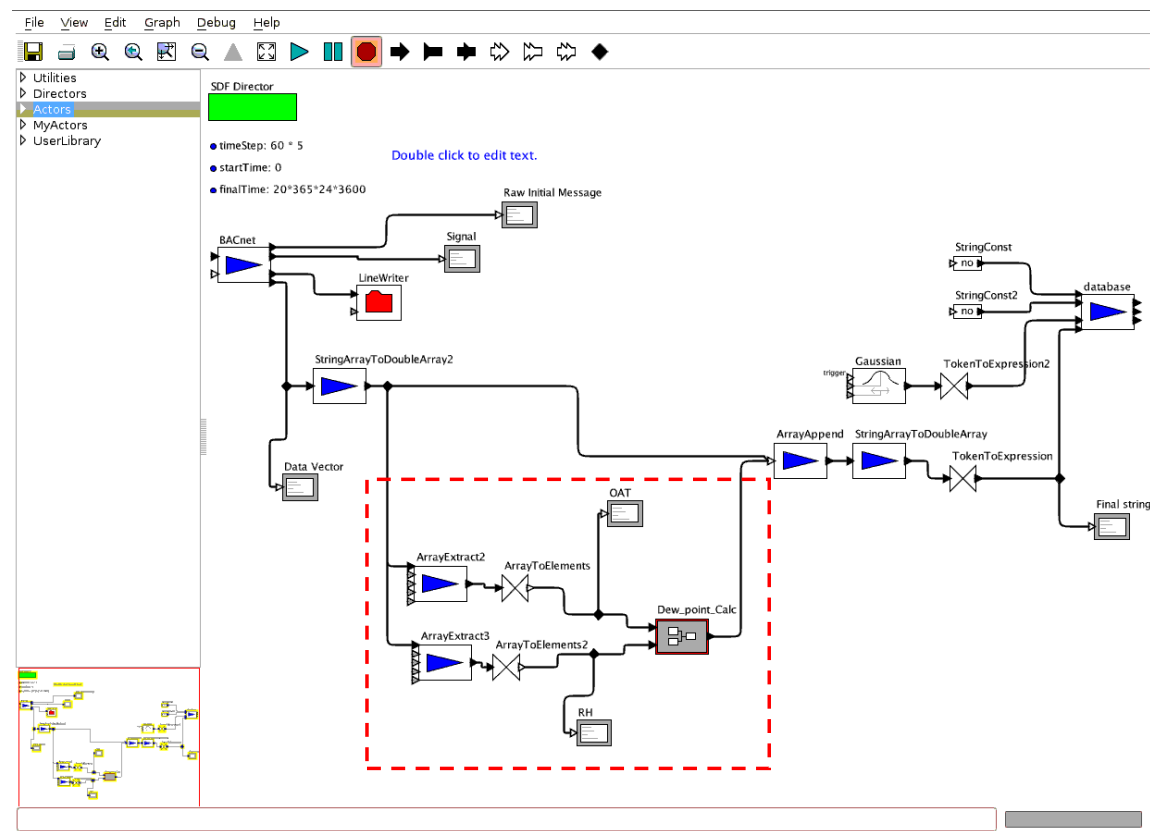

Figure 3 Graphical programming of the BACnet read process using the Ptolemy II GUI

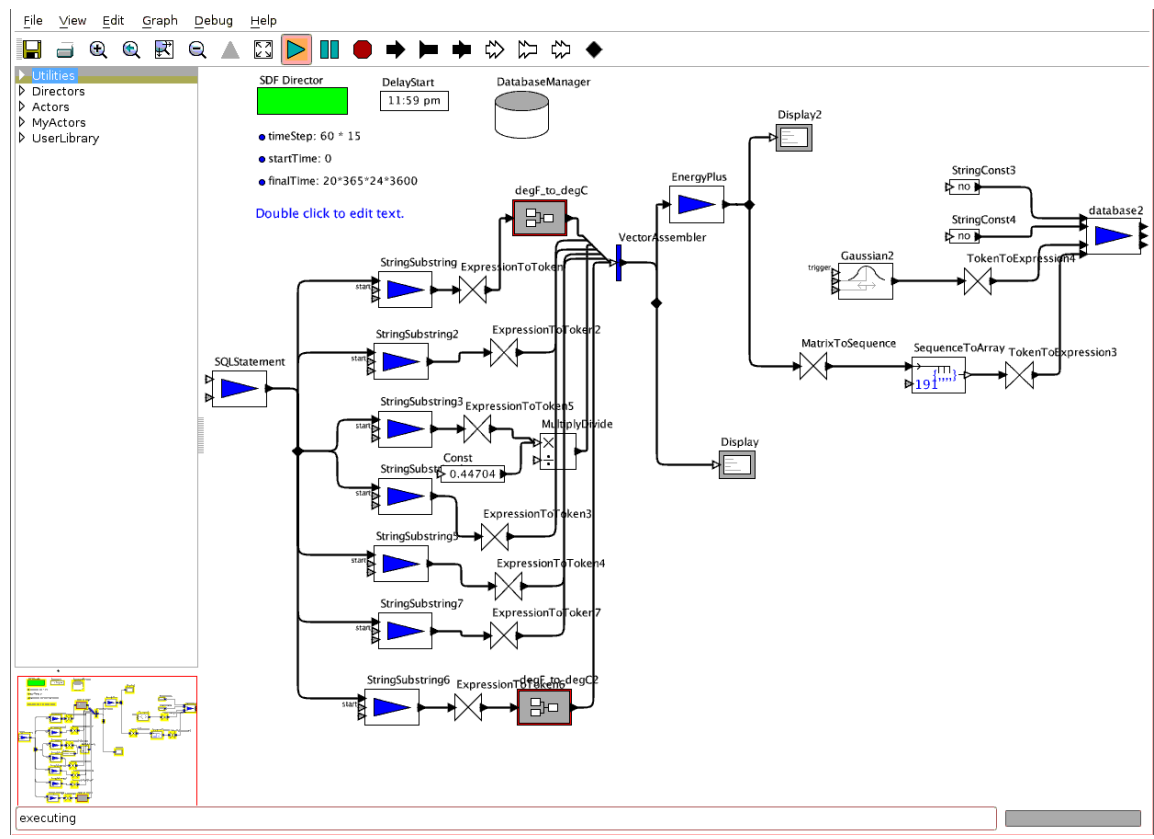

Figure 4 Graphical programming of EnergyPlus simulation using the Ptolemy II GUI

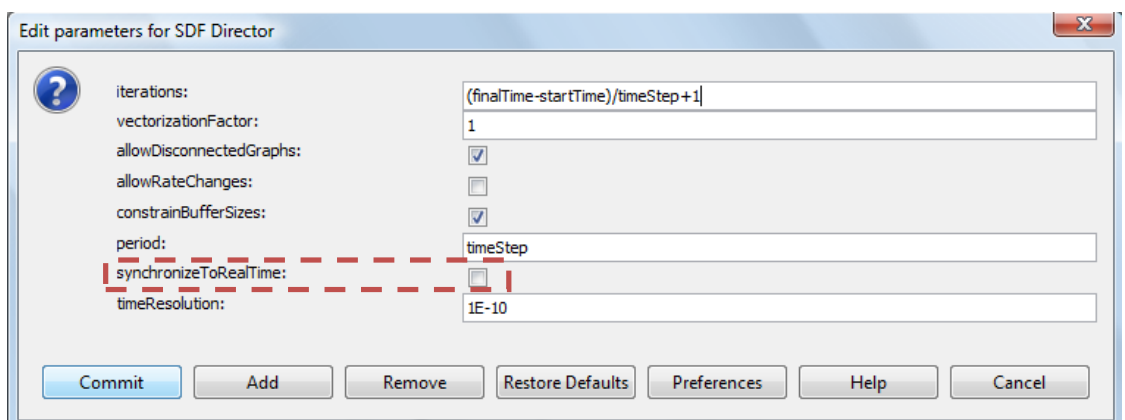

Figure 5 Enable synchronization of simulation time to real time 


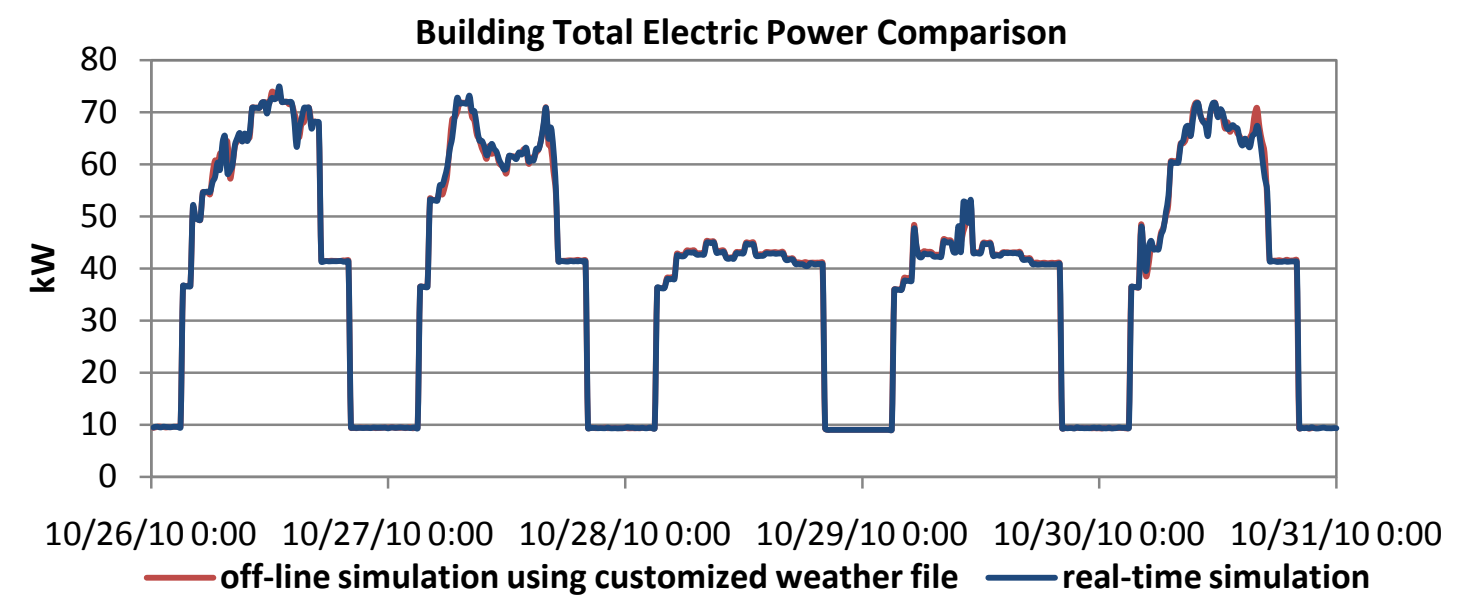

Figure 6 Building total electric power comparison between real-time simulation and off-line simulation using the customized weather file

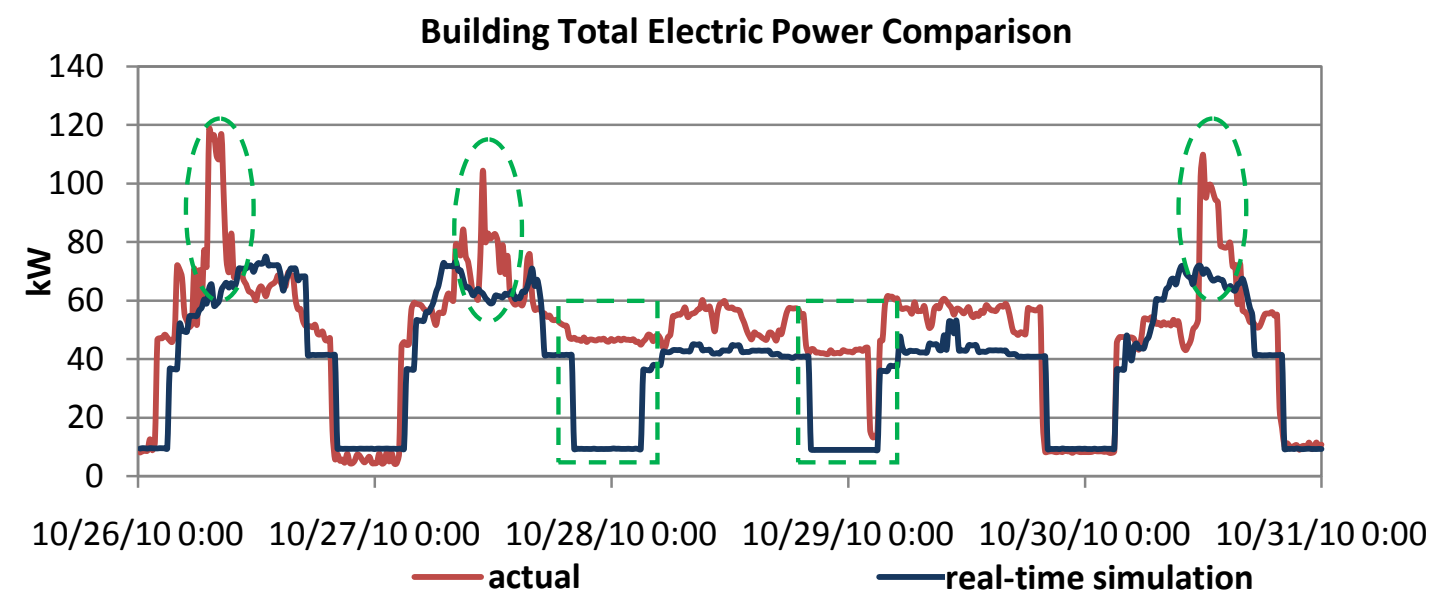

Figure 7 Building total electric power comparison between real-time simulation and actual measurement

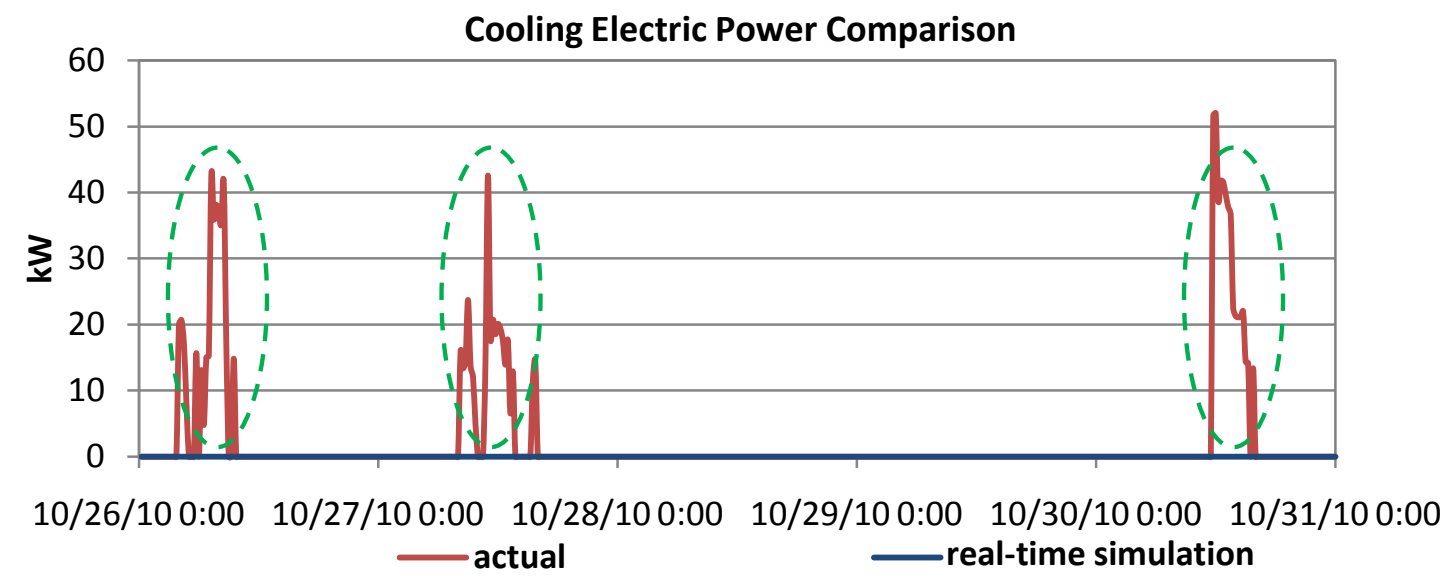

Figure 8 Cooling electric power comparison between real-time simulation and actual measurement 


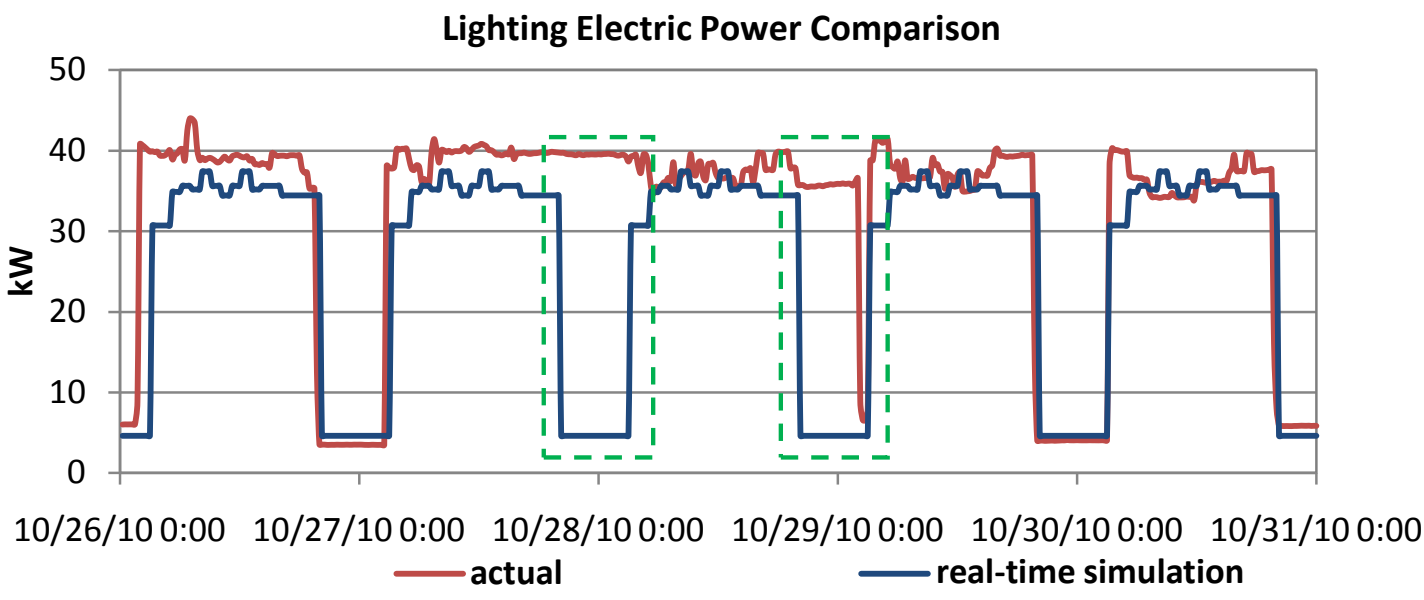

Figure 9 Lighting electric power comparison between real-time simulation and actual measurement 